\title{
Motor Drive Module Design for Electric Care Bed
}

\author{
Peng Yang*, Shujuan Zhu \\ Hebei University of Technology, Tianjin 300130, China \\ CNR Tangshan Railway Vehicle Co., Ltd, Tangshan 063035, China \\ * Corresponding authors Email: moonlight034142@126.com
}

\begin{abstract}
The electric care bed control system is divided into three modules, which are physiologic parameter detection module, human-computer interaction module and motor drive module (MDM). Those modules communicate with each other by controller area network (CAN) bus. Under the idea of modular design, a plug and play (PnP) multifunctional motor drive module (MDM) is developed. This MDM has a CAN interface which can make it possible to communicate with human-computer interaction module, and then control the position closed-loop and speed closedloop. The MDM based on CAN bus will be more efficient in communication and system expansion and make the electric care bed function perfectly.
\end{abstract}

Keywords: electric care bed; motor drive module (MDM); controller area network (CAN) communication; position control; speed control

\section{Introduction}

Nowadays, the worldwide population of elderly people is growing rapidly and in the coming decades the proportion of elderly people will change significantly. This demographic shift creates a huge increase in the demand for health-care services. So it is necessary to develop an electric care bed to satisfy the requirements of elderly people and the patients with chronic disease. Besides, in the market, the electric care bed is popular. The top products have high price and big investment. But some medium and low quality products can only complete the moving of back and bending of legs by common mechanical structure and drive mechanism. Therefore, it is necessary to develop an electric care bed based on modular design and with features of simple operation, high intelligence, complete functions, safety and comfort ability. This paper introduces a high-speed and high-accuracy motion control system for electric care bed, the system has high integration and performance, expanding and with good

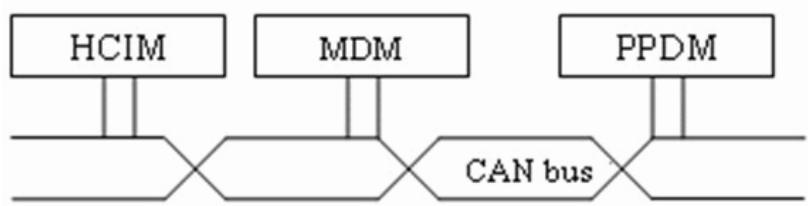

Figure 1. Modules Interconnection on CAN Bus Diagram

universality.

With the concept of modularization, the electric care bed control system is divided into three modules, which are physiologic parameter detection module (PPDM), human-computer interaction module (HCIM) and MDM. Figure 1 is an interconnect module block diagram on CAN bus.

In the general control system, some modules exchange amount of data, such as position and speed signals and some physiological parameters to work. Therefore, it is extremely important to exchange data between modules accurately and real-timely. Most control systems use RS-232 or RS-485 bus whose com- 


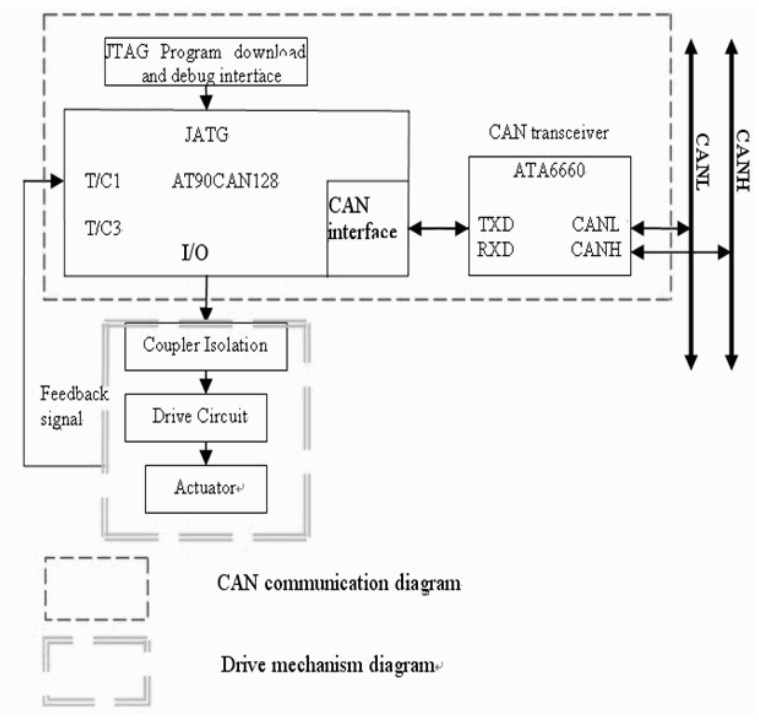

Figure 2. Motor Drive Intelligent Node's Block Diagram

munication means is the command and response mode. That is the host sends an inquiry to sub-controllers, and then sub-controllers upload the data of their own status. The shortcoming of this approach is low data transmission efficiency. As the host controller is busy, the sub-controller can't upload data immediately and has to wait for an order from the host. To overcome the above shortcoming, three modules are combined into an intelligent network through CAN bus.

Under the idea of modular design, a PnP (plug and play) multifunctional MDM is developed. This MDM has CAN interface that makes it possible to communicate with HCIM, and then achieves the position closedloop and speed closed-loop.

\section{Intelligent $\mathrm{CAN}$ node-MDM}

In this system, three modules are combined into an intelligent network through CAN bus. Each module connects through CAN bus and among the modules there are multi-host communication relationships. So, each module is equipped with CAN interface, which makes them exchange data between each other. Figure 2 is motor drive intelligent node's block diagram.

Human-computer interaction module controls the operation of actuator through CAN bus. Also the MDM transmits real-time speed and position information to human-computer interaction through CAN bus. In order to adjust the position of body, the MDM use AT90CAN128 as the controller, and then it can rapidly and effectively control the action of linear actuator. The actuator drives the electric care bed's motion mechanism, and then the function of electric care bed is completed. In this paperthe CAN bus interface design and MDM speed and location of closed-loop control are mainly introduced.

The actuator has complex mechanism to transfer rotary motion to linear motion. And it has the following features such as damping and noise-reducing, fullclosed and humidity resistance design. In the process of push-pull, the linear actuator has an internal limited switch in order to prevent actuator from locked, and then leads speed to zero; electric current is too large to burn motor boards.

Using Hall sensor (3144) as the feedback component, these Hall-effect switches are monolithic integrated circuits with tighter magnetic specifications are more stable with both temperature and supply voltage changes. The unipolar switching characteristic makes these devices ideal for use with a simple bar or rod magnet. Hall sensor is identical except for magnetic switching points. Each device includes a voltage regulator for reverse battery protection diode, quadratic Hall-voltage generator, temperature compensation circuitry, small signal amplifier, Schmitt trigger, and with suitable output pull up, they can be used with bipolar or CMOS logic circuits.

\section{CAN bus interface design}

\subsection{CAN bus communication hardware design}

CAN bus, which supports distributed control or realtime control, is a serial controller area network. CAN communication hardware structure is shown in figure 1. The hardware mainly includes single chip microcontroller AT90CAN128, CAN bus transceiver ATA6660 and high-speed optocoupler 6N137, etc.

AT90CAN128 is a low-power CMOS 8-bit microcontroller based on the AVR enhanced RISC architecture. By executing powerful instructions in a single clock cycle, the AT90CAN128 achieves throughputs approaching 1 MIPS per MHz allowing system designers to optimize power consumption versus processing speed. The AT90CAN128 CAN controller is fully compatible with the CAN Specification 2.0 Part $\mathrm{A}$ and Part B. It delivers the features required to implement the kernel of the CAN bus protocol according to the ISO/OSI Reference Model.

ATA6660 is a high-speed CAN transceiver. It is especially designed for high speed CAN Controller differential mode data transmission between CAN-Controllers and CAN bus. It is fully compatible to ISO11898. In normal case the RS pin connects to ground directly, so baud rate can be adjusted up to $1 \mathrm{M} / \mathrm{s}$. 


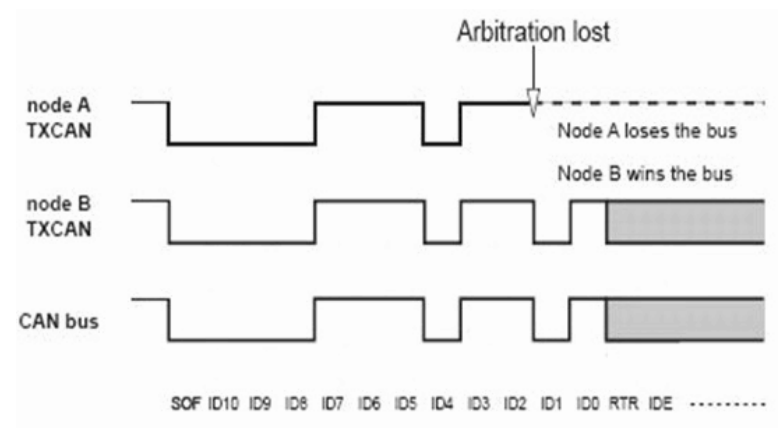

Figure 3. Bus Arbitration

Considering the anti-interference ability of CAN bus AT90CAN128 is not directly linked to the ATA6660, but through the high-speed and performance optocoupler $6 \mathrm{~N} 137$ that is good for electrical isolation between the CAN nodes and can speed up to $10 \mathrm{Mb} / \mathrm{s}$.

In the hardware circuit design, AT90CAN128 with $8 \mathrm{MHz}$ crystal frequency is mainly responsible for the initialization of CAN communication, receiving-transmitting messages. As a result of on-chip CAN controllers, the communication hardware circuit design can be greatly simplified and system reliability can be enhanced.

\subsection{CAN bus communication software design}

This section uses the integrated designer environment AVR Sutdio4 and compiler AVR-GCC. The software development environment is provided by Atmel Company. In communications, CAN-usb interface card and CAN test software ZLGCAN Test are provided by ZhouLiGong Company.

\subsubsection{The design of CAN protocol}

The CAN protocol handles bus accesses according to the concept called "Carrier Sense Multiple Access with Arbitration on Message Priority" (shown in Figure 3).

During transmission, arbitration on the CAN bus can be lost to a competing device with a higher priority CAN identifier. This arbitration concept avoids collisions of messages whose transmission was started by more than one node simultaneously and makes sure the most important message is sent first without time loss.

The bus access conflict is resolved in the arbitration field mostly over the identifier value. If a data frame and a remote frame with the same identifier are initiated at the same time, the data frame prevails over the remote frame.
In this system, CAN bus communication nodes is a multi-host, that is, any node can not only transmit messages to other nodes, but also receive messages from other nodes through CAN bus. Thus, each node needs to allocate its own ID. Nodes distribution is shown in Table 1.

Table 1. CAN Nodes ID distribution

\begin{tabular}{|c|c|c|c|c|c|c|c|c|c|}
\hline No. & ID & ID & ID & ID & ID & ID & ID & ID & Node \\
& 12 & 11 & 10 & 9 & 8 & 7 & 6 & 5 & name \\
\hline 0 & 0 & 0 & 0 & 0 & 0 & 0 & 0 & 1 & MDM \\
\hline 1 & 0 & 0 & 0 & 0 & 0 & 0 & 1 & 0 & HCIM \\
\hline 2 & 0 & 0 & 0 & 0 & 0 & 0 & 1 & 1 & PPDM \\
\hline 3 & \multicolumn{10}{|c|}{ Reserved } \\
\hline
\end{tabular}

\subsubsection{The program design of CAN communica- tion}

CAN bus communication is the basic part of the motor driving module software design, mainly including: receiving and processing messages from other nodes on CAN bus; sending the state news of this controller. CAN communication module includes CAN initializing program, data receiving procedure and data sending procedure.

The following mainly describes the initializing program of CAN bus and the data transmission program.

CAN bus initial function: can_init (U8 mode) is used for some parameters of CAN bus, such as the bus baud rate initialized, empty CAN message box, and CAN bus enabled, etc.

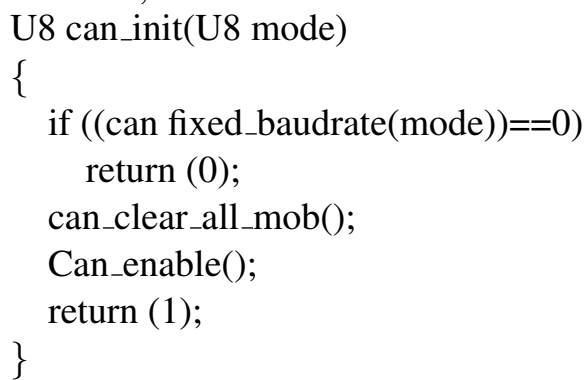

The procedures of CAN bus transmission program are as followsinitialize the following fields before sending: Identifier tag(IDT), Identifier extension (IDE), Remote transmission request (RTRTAG), Data length (DLC), Reserved bit tag (RBNTAG), Data bytes of message (MSG); set the message boxe in Tx configuration for the Mob to send data or a remote frame; then the CAN channel scans all the message box in Tx configuration, seeking for the message box having the highest priority and try to send it. All the parameters and data are available in the message box until a new initialization. The flowchart of AT90CAN128 sending program is shown in Figure 4, 


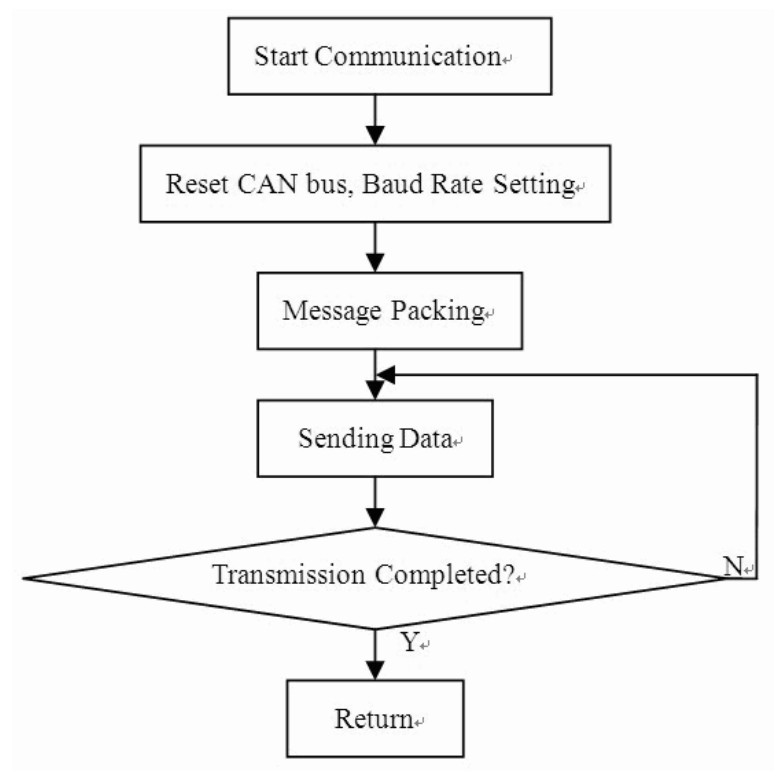

Figure 4. Flowchart of CAN Bus Transmission

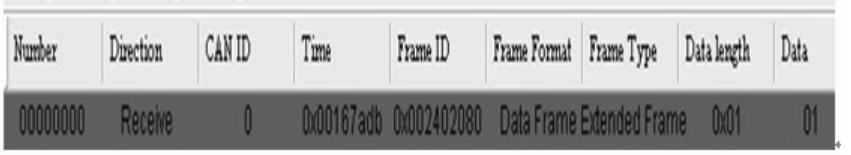

Figure 5. Executive Results

CAN bus transmission program is listed as follows: void can_TX(void)

\{

message.id.std $=0 \mathrm{x} 0 \mathrm{~F}$;

message.pt_data $=$ \&buffer[0];

message.ctrl.ide $=0$;

message.dlc $=1$;

message.cmd $=$ CMD_TX_DATA;

while(can_cmd(\&message)!=CAN_CMD_ACCEPTED);

while(1)

\{

\}

if $\left(\mathrm{u} 8 \_\right.$temp $==$CAN_STATUS_ERROR)

$\mathrm{b}=1$;

Through CAN interface card, PC sends command frame. The single-chip receiver enables the motor to implement corresponding movements and send the appropriate message in response to the CAN card, after sending the appropriate message in response to the bus, while the PC machine receives messages and display on the screen through the CAN debugging software ZLGCAN Test. The executive results are shown in Figure 5.

Figure 5 shows that the received frame identifier ID is 0x02402080. The Destination address is the user's input unit, the source address is the 1st motor execution unit, the data length is 1 , and the value is $0 \times 01$,

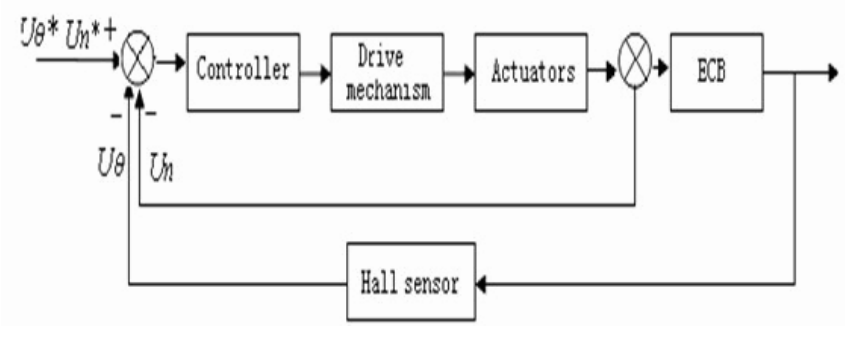

Figure 6. Motion control system Design of Electric Care Bed

representing that the MDM has received an order entered by HCIM.

\section{Closed-loop motion control system design for electric care bed}

The motion control system for electric care bed has high integration, perfect function and user friendly interface. Three modules are combined into an intelligent network through CAN bus. In order to adjust the position of body, AT90CAN128 is used to rapidly and effectively control the action of linear actuator. The actuator drives the electric care bed's motion mechanism, and then the function of electric care bed is completed.

Closed-loop motion control system is composed of a controller, a sensor, a drive mechanism and actuators. Figure 6 is the closed-loop motion control system design of electric care bed.

In Figure $6, U_{\theta}^{*}$ and $U_{n}^{*}$ represent position set value and velocity set value. $U_{\theta}^{*}$ and $U_{n}^{*}$ represent position feedback value and velocity feedback value respectively. Double closed loop control is implemented with Hall sensor through signal of speed and position.

Based on the software and hardware development platform, using the sensor and microcontroller technology, users can use the keyboard to control the electric care bed to get the most comfortable position and remember it. At the same time, users can control the speed with control handle and set different speed according to their different requirements. In this way, the care bed design can achieve the MDM position and speed control.

\subsection{Speed control of motion control system}

Speed control is achieved through pressing the up/ down buttons to adjust the speed. Press the button (OK), the speed can be stored. If the speed needs to be modified, press the "reset" button. In addition to these basic speed control functions, the following features like acceleration or deceleration can also be 


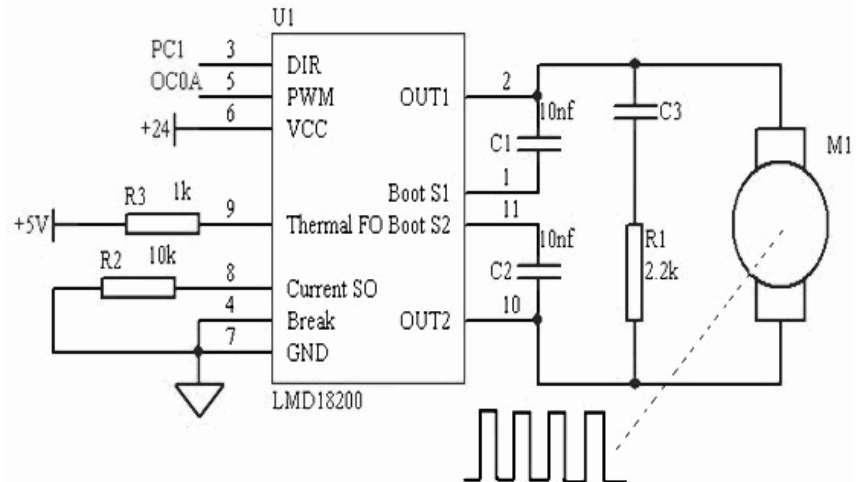

Figure 7. AT90CAN128 and LMD18200 Interface Circuit AT90CAN128 receives speed and position

added. AT90CAN128 regulates PWM value to control the actuator.

\subsubsection{The design of speed control circuit}

The LMD18200 is a 3A H-Bridge designed for motion control applications. The device is built to use a multi-technology process which combines bipolar and CMOS control circuitry with DMOS power devices on the same monolithic structure. Being ideal for driving DC and stepper motors; the LMD18200 accommodates peak output currents up to 6A and operates at supply voltages up to $55 \mathrm{~V}$.

There are four timers / counters (16 bit T/C1 and $\mathrm{T} / \mathrm{C} 3,8$ bitT/C0 and T/C2). Timer/Counter0 is a general purpose, single channel, 8-bit Timer/Counter module. The 16-bit Timer/Counter unit allows accurate program execution timing (event management), wave generation, and signal timing measurement. Timer/Counter2 is a general purpose, single channel, 8-bit Time$\mathrm{r} /$ Counter module. The 16-bit timer / counter can generate 10 independent interrupts, which adjust the PWM cycle. The 16-bit timer / counter with input capture unit which can be applied to record the external incident as well as the time it has been lasted.

AT90CAN128 and LMD18200 form a unipolar driving closed-loop DC motor control circuit as shown in Figure 7. In this circuit, the PWM controls signal input to pin 5 (connect with OC0A of AT90CAN128), turns signal input to pin 3 (connect with PC1 of AT90CAN128). The speed and direction are controlled by regulating PWM duty cycle. The position of actuator is obtained by detecting the feedback signal of Hall sensor, which forms the position closed loop control to achieve precise control of actuator. feedback signal, and regulates PWM waveform by $\mathrm{T} / \mathrm{C} 0$, and then the actuator achieves a constant speed. The PWM resolution for the phase correct PWM mode is fixed to

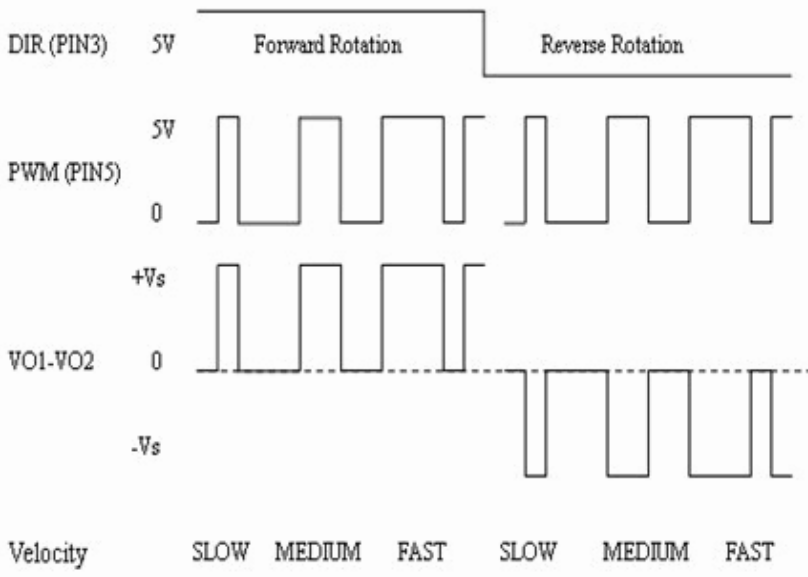

Figure 8. Ideal PWM Waveform

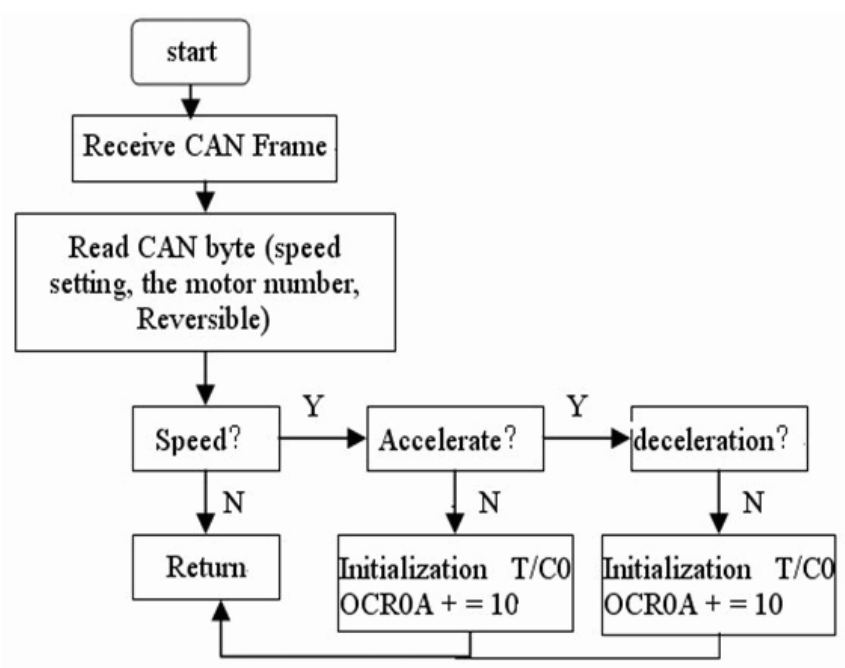

Figure 9. Flowchart of Speed Control Initialization

eight bits. In phase correct PWM mode, the counter is incremented until the counter value matches MAX. When the counter reaches MAX, it changes the count direction. The TCNT0 value will be equal to MAX for one timer clock cycle. Figure 8 is the ideal unipolar PWM waveform.

\subsubsection{The software design of speed control}

AT90CAN128 receives the message through the CAN bus, and then controls the motor to start and stop, in the forward rotation acceleration / deceleration, and in the reverse acceleration / deceleration. This control set the motors' speed and directions mainly through receiving the display module's message. The speed control initialization flowchart is shown in Figure 9. Obtained pulse frequency through the input capture interrupt, the program is as follows: 


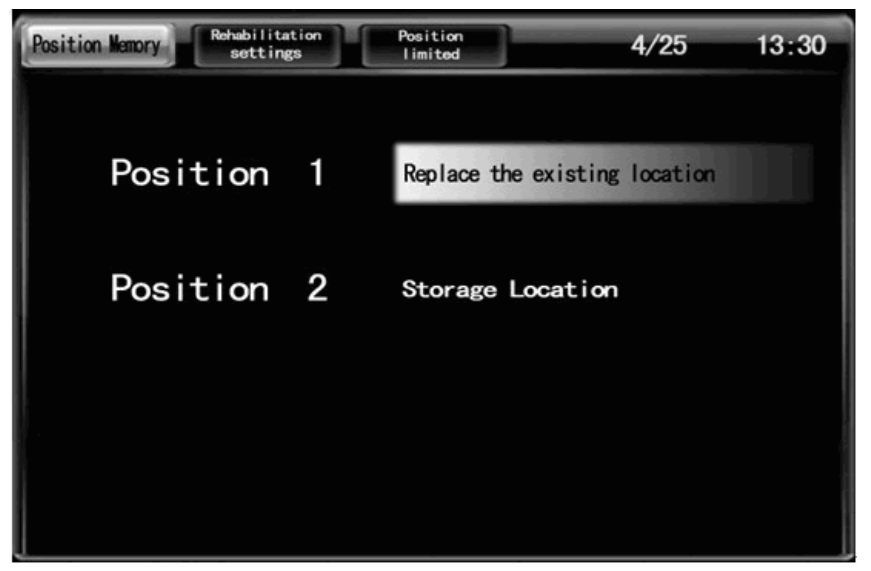

Figure 10. the Interface of Position Selection

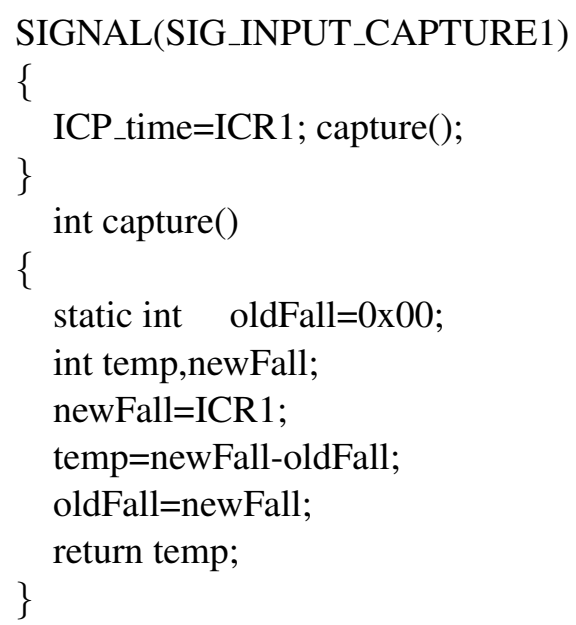

\subsection{Position control of motion control system}

Some products using electric motors are not only limited in the effect of start and shut control and direction control, but also in short of the location control or speed control. The following mainly introduces the position control principle of the linear actuator mechanism with position sensor. The position memory function can be applied to many of the controlled objects which do not require high accuracy such as electric curtain, electric doors, electric seats, etc.

Position control design can simplify the user's operation. Users can operate the bed to the most comfortable position, and then click "Position Memory key" to store the position. If users want to achieve this position again, click "Location 1" key, the actuator will push bed to the last set position. For users, the position memory function can remember two positions by pressing the "position 1", "Location 2" key. If users want to adjust the set position, just press "location memory" button, the user interface will appear (as shown in Figure 10).

\subsubsection{The hardware design of position control}

1. Absolute position control hardware design

Absolute position control is the benchmark running position of electric care bed. Using the Hall sensor as reference position detection feedback device, the output of these devices (Pin 3) switches low when the magnetic field at the Hall sensor exceeds the operating point threshold. At this point, the output voltage is VOUT. When the magnetic field is reduced below the releasing point threshold, the device output goes high. The difference between the magnetic operating and releasing points is called the hysteresis of the device. This built-in hysteresis allows clean switching of the output even in the presence of external mechanical vibration and electrical noise.

Hall sensor works as follows: When current I on the magnetic field goes through the Hall sensor, if the current direction is perpendicular to the magnetic field, the horizontal side of the semiconductor substrate, which is between vertical sub-current and magnetic flux, generates a voltage. The voltage is called Hall voltage UH. Hall voltage is proportional to the current (I) and magnetic induction (B), as shown in Eq. (3.1):

$$
U_{H}=R_{H} I B / d
$$

Where $R_{H}$ is the hall coefficiency, $d$ is the thickness of the substrate, $I$ is the electric current, $B$ is the magnetic induction. The current is constant and the Hall voltage is proportional to the magnetic induction $(B)$, sizing only to the size of the magnetic induction, and has nothing to do with flux change rate. In this system, there are four Hall sensors to control the electric care bed's four postures. The sensor's hardware design is shown in Figure 11. Pin 1 is connected to $+5 \mathrm{~V}$, to supply the voltage. Pin 2 is connected to GND. Pin 3 is connected to the external interrupt pin of AT90CAN128(PD0,PD1,PD2,PD3).

\section{Relative position control hardware design}

The linear actuator with the position sensor, putting retractable rod a lead, while the linear actuator gives six pulses, through the controller unit to capture the pulse count, and then control the position of electric care bed. The fol- 


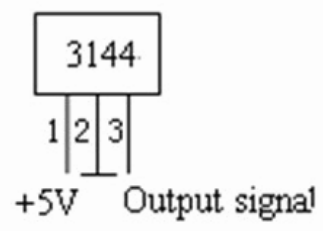

Figure 11. Hardware Design of Hall Sensor

lowing introduces the interface between actuator and drive circuit (shown in Figure 12).

In the picture, the interface has four pins, as shown in Figure 9, signal 1 and signal 2 being two outputs of internal position sensor. While the sensor circuit in the actuator is OC gate, so it is necessary to build a pull-up resistor in peripheral circuit of the internal position sensor, and then Signal 1 output pulse signal (shown in Figure 13).

AT90CAN128 collects output pulses and calculates the number, frequency, comparing with the predetermined value, and then controls the actuator to reach the corresponding position and velocity. Thus, it achieves electric care bed's position and speed control.

\subsubsection{The software design of position control}

Position control is divided into two parts, one being absolute position control, and the other being the relative position control.

\section{Absolute position control}

To control the position of MDM, reference position should be confirmed. Reference position is designed to achieve through the Hall sensor, using four Hall sensors to control the reference position of back's lifting/falling, leg bending and body turning over.

Horizontal position detection (Reference position detection) principle: The Hall sensor is a

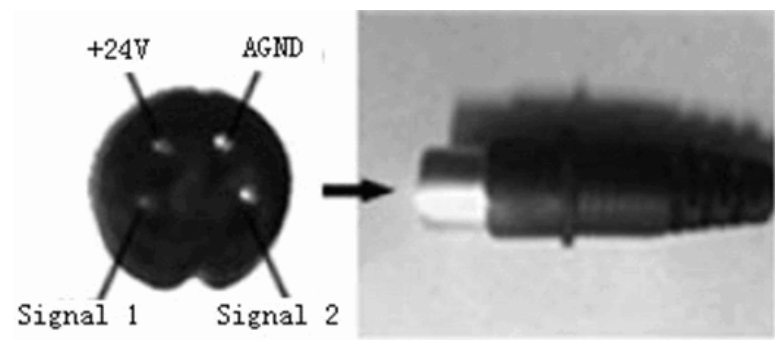

Figure 12. the Picture of Actuator Interface

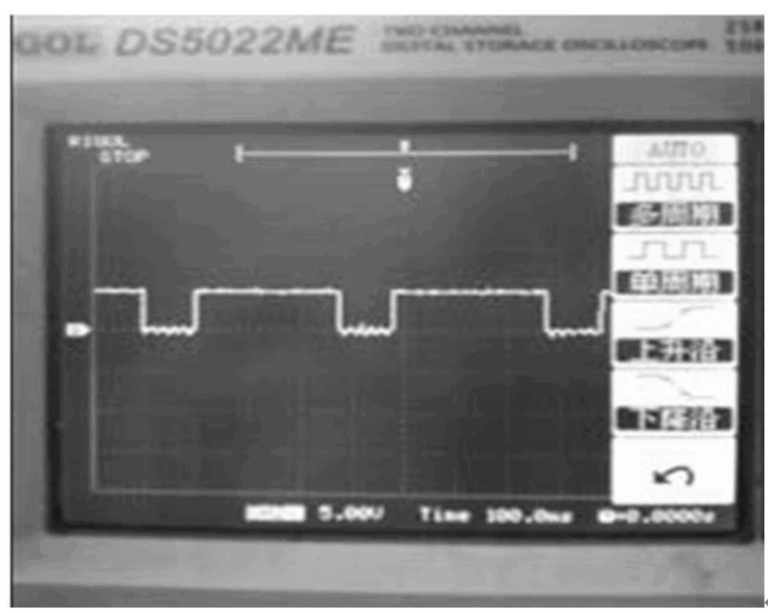

Figure 13. the Picture of Output Pulse Signal

magnetic sensor. When the S-pole of magnet closes enough, the hall sensor output is low level, otherwise the output is high level. The sensor's output signal input AT90CAN128 the INT0, INT1, INT2, INT3 four external interrupts (Pin PD0,PD1,PD2,PD3), and the falling edge trigger the interrupt.

The external interrupt initialization functions as follows:

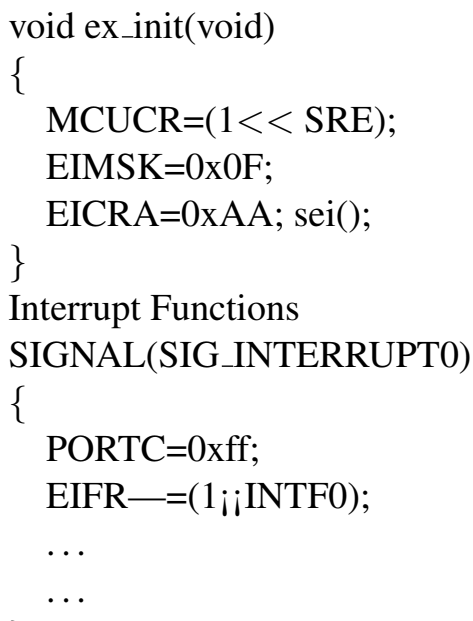

2. Relative position control

Comparing position control to speed control, there are two differences: The motor should be able to achieve positive/reverse rotation. This has been resolved in the design of drive circuit. Once it reaches the set value, the motor will stop. The problem should be resolved by detecting feedback signal. Feedback pulses generate from the position sensor of actuator itself. The signal inputs to ICP1. When the logic level on the external pin ICP1 changes and has con- 


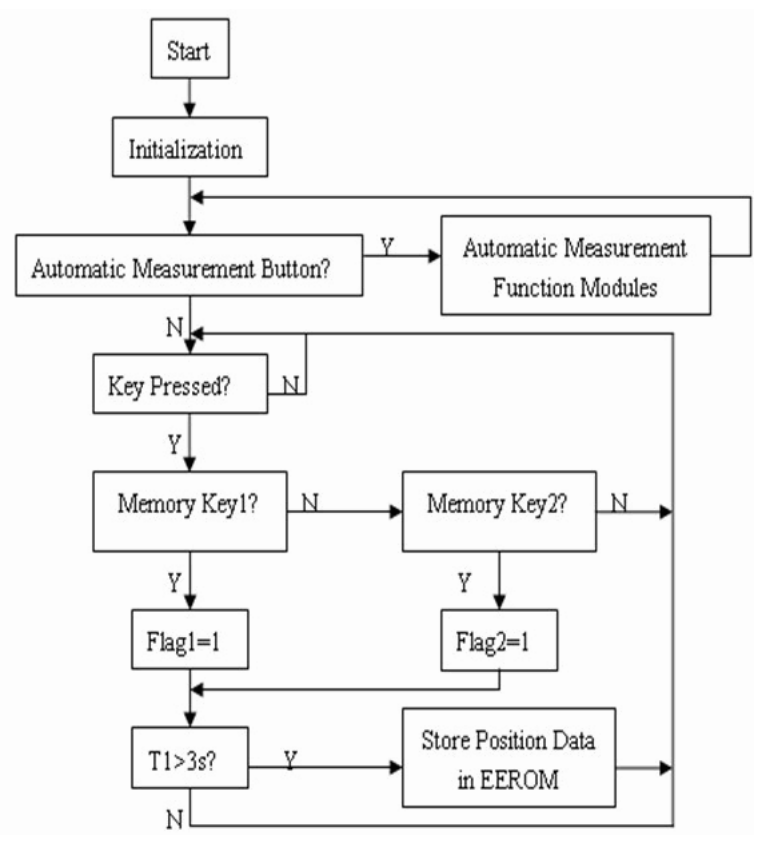

Figure 14. Flowchart of Position Memory Subroutines

firmed by edge detector, the input capture is inspired. At this time the value in TCNT1 is written to ICR1 and set ICF1. Interrupt at this time is triggered through disruption of routines to achieve the control of motor.

For position control of MDM, it is primary to design limited position of motor's movement, and as a benchmark. Controller stores pulses when actuator achieves the limited position, that is, actuator's benchmark in the direction.

Principle in calculating and memorizing location: there are manual buttons and store buttons on portable keyboard. The manual buttons can control the linear actuator to achieve a appropriate location, while the single-chip calculates feedback pulses, and then if press the storage key more than 3 seconds $(t 1 \geq 3)$, this location information will be stored into the single-chip and memory function will have been completed (shown in Figure 14). Users can set two comfortable positions according to their own requirements.

\section{Conclusion}

This paper describes the hardware and software design of CAN bus communication and the position and speed control design of electric care bed's MDM. The communication among MDM and other intelligent modules makes full use of the character of CAN bus that it can transmit and receive message accurately, realtimely and effectively. Under the idea of modular design, a PnP multifunctional MDM is developed. MDM can communicate with human- computer interaction module through CAN bus, and then control the position closed-loop and speed closed-loop. This design can make the electric care bed function perfectly.

The intelligent motor drive module can be expanded to other system with CAN nodes. Memory function is the expansion of position control. Due to the existence of memory function, the MDM can be used into some systems which need position control. The intelligent MDM enables operation simpler and convenient. The MDM based on CAN bus has not only executive functions, but also control and communication functions. The module can be applied to some systems to improve the extensibility and real-time performance of them. So the MDM will have better application and development value.

\section{Acknowledgments}

This work was supported by the National Key Technology R\&D Program under Grant No. 2006BAI22B03.

\section{References}

[1] Hande Alemdar, Cem Ersoy. Wireless sensor networks for healthcare: A survey. Comput. Netw. 2010.

[2] Diaz, J., Rodriguez, E., Hurtado, L., Cacique, H., Vazquez, N., Ramirez, "A. CAN bus embedded system for lighting network applications Circuits and Systems", MWSCAS 2008. 51st Midwest Symposium on 10-13 Aug, 2008: 531 - 534.

[3] Huiqian Yang, Yaonan Wang, Xiaofang Yuan. "Development of Data Acquisition and Display System of HEV CAN BUS", Computer Engineering and Applications, Vol.20 2006: 228-232.

[4] Jiying Li. "Research on Frequency-shift Send Equipment with Microcomputer", Journal of Lanzhou Jiaotong University (Natural Sciences Edition), Vol.26, No.1, 2007: 32-34.

[5] Michael A.Goodrich, Alan C. Shultz. "A survey Foundations and Trends in Human-computer Interaction". Human-robot interaction. Vol.14, No.2, 2007.

[6] Atmel. AT90CAN128-Datasheet-Revg, USA, Atmel Corporation, 2007.

[7] Paolo Dario, Eugenio Guglielmelli, Vincenzo Genovese, Maurizio Toro. "Robot assistants: Applications and evolution". Robotics and Autonomous Systems, Vol.18, No.3, 1996: 225-234. 